\title{
Experimental Investigation of the Aerodynamic Flow in the Aircraft Carrier Ski-jump by means of PIV
}

\author{
R. Bardera ${ }^{1}$, M. León-Calero² and A. A. Rodríguez-Sevillano ${ }^{3}$ \\ 1. Instituto Nacional de Técnica Aeroespacial (INTA). Ctra. Ajalvir, km 4.5.Torrejón de Ardoz, 28850 Madrid, Spain
}

Email: $\underline{\text { barderar@inta.es, web page: http://www.inta.es }}$

2. Instituto Nacional de Técnica Aeroespacial (INTA). Ctra. Ajalvir, km 4.5. Torrejón de Ardoz, 28850 Madrid, Spain

Email: leoncm@inta.es,web page: http://www.inta.es

3. Escuela Técnica Superior de Ingeniería Aeronáutica y del Espacio. Universidad Politécnica de Madrid (UPM). Plaza del

Cardenal Cisneros, 28040 Madrid, Spain

Email: angel.rodriguez.sevillano@upm.es,web page: http://www.upm.es

\begin{abstract}
Computational Fluid Dynamics (CFD) methods have opened a new field to perform aerodynamic studies saving money and time. The difficulties presented by this method to calculate complex flow field problems imply that CFD validation is needed to provide correct results. Experimental data have recently been used to validate the accuracy of CFD predictions. Particle Image Velocimetry (PIV) has shown to be a powerful tool in the investigation of complex flows. The aim of this paper is to present results from PIV experiments that would be interesting for CFD validation.

Regarding aircraft operations, the short runway available implies the necessity of equipment which helps to take-off performances. Ski-jump ramp system improves aircraft performances by an increment of lift resulting in successful take-off operations. The ski-jump ramp presence generates a complex flow bounded by a turbulent shear layer and a low velocity recirculation bubble over the end of the flight deck. The adverse effects on the aircraft aerodynamics affect to pilot safe operations, so this region is an interesting problem to be studied by means of wind tunnel experimental tests.
\end{abstract}

Key words: Experimental aerodynamics, PIV, CFD validation, ski-jump ramp 


\section{Introduction}

Aircraft carriers are essential for military operations, providing tactical advantages to support maritime tasks far from their country [1,2]. Despite routine operations, pilots have to deal with challenging environments [3, 4]. The structure of aircraft carriers is designed with practical rather than aerodynamic considerations [5]. Moreover, the superstructure is essentially a combination of bluff bodies [2,6] with sharp edges and corners, antennas, stacks, radomes... that generate a very complex flow field $[4,7]$. Hence, pilots must fly through a highly turbulent flow structure [2].

Flow field configuration is affected by wind direction, ship motion and aircraft operational attitude $[1,2]$. Regarding that helicopter operations can be performed at different conditions [8], the flow encountered by the helicopter near or over the flight deck is highly complex and unsteady [9, 10]. Crosswind direction is the critical one due to the fact that the massive flow detachment generates a turbulent shear layer and a low velocity recirculation bubble over the flight deck $[1,2,11]$. Consequently, pilots have to handle with workload increment $[4,12]$ and low safety levels during on board operations [13].

Regarding airplane take-off manoeuvre, the short runway available implies the necessity of equipment which helps to operate in these conditions. Ski-jump ramp system improves aircraft performances by an increment of lift resulting in successful take-off operations [14]. Ahead wind condition is the common configuration during take-off. The ski-jump ramp presence at the end of the flight deck changes the flow structure, so aircraft have to deal with non-stationary aerodynamic effects caused by a turbulent shear layer which delimits the recirculation bubble region [11].

The study of this problem has become more common by using wind tunnel tests [15] and computational methods [16]. Previous researches developed at INTA showed the adverse aerodynamic effect generated in this ship model at ahead wind conditions in the central section of the ski-jump ramp [15].
Advances in Computational Fluid Dynamics (CFD) methods have opened a new field to perform aerodynamic studies that save money and time. The difficulties presented by this technique to calculate complex flows mean that CFD validation is needed in order to provide correct results [13, 17, 18]. Experimental data are continuously used to validate the accuracy of CFD predictions [7, 19].

In the last decades, Particle Image Velocimetry (PIV) has shown to be a powerful tool in the investigation of complex flows [20] to obtain insight about flow during wind tunnel experimental simulation. In addition, PIV shows to be a useful technique for CFD validation [21].

In the present paper, the flow field in the vicinity of a ski-jump ramp has been investigated by testing a 1:100 scaled model of an aircraft carrier. The test experiments were performed in the large wind tunnel INTA-T1 of Instituto Nacional de Técnica Aeroespacial (INTA). PIV technique was used to measure the flow field velocity in the region of interest. The aim of this paper is to present PIV results that play a fundamental role to validate numerical simulations by means of CFD numerical codes.

\section{Wind Tunnel Set Up}

\subsection{Wind Tunnel}

The experiments were carried out in a low-speed wind tunnel at the facilities of Instituto Nacional de Técnica Aeroespacial (INTA), located in Madrid (Spain). The wind tunnel INTA-T1 has a closed-circuit and an open test section of $2 \times 3 \mathrm{~m} 2$. The power supply system consists on a DC $450 \mathrm{~kW}$ electric motor at 420 $\mathrm{V}$, which provides a maximum freestream velocity of $60 \mathrm{~m} / \mathrm{s}$ and a turbulence intensity lower than $0.5 \%$.

Figure 1 shows the aircraft carrier model located in the test section oriented with an incident wind angle of $0^{\circ}$. The ship model is located over a platform that simulates the sea surface. The interference between the platform and the incident flow field is reduced by using streamlined leading and trailing edges [22]. 
Regarding sharp-edged structures, experimental test conditions for a Reynolds number higher than the critical Reynolds number (typically $1.1 \times 104$ ) ensures similarity between the model and the full-scale aircraft carrier [23]. Hence, this condition is fulfilled for a wind tunnel velocity of $10 \mathrm{~m} / \mathrm{s}$ at ahead wind direction for a Reynolds number of $1.3 \times 105$ based on the height of the ramp.

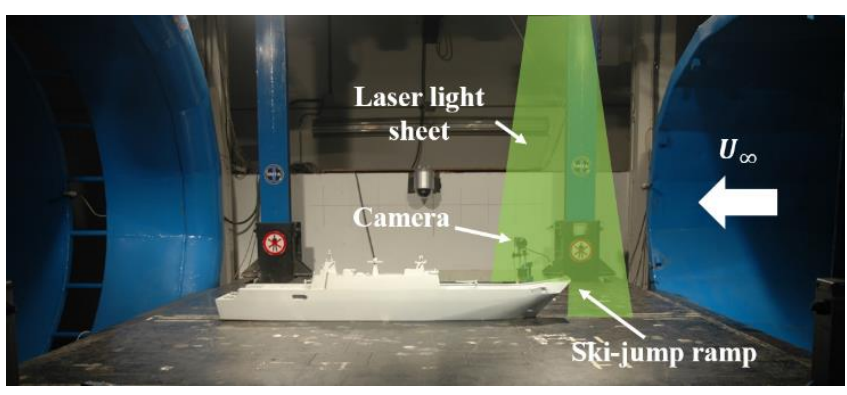

Figure 1: Ship model located in wind tunnel test section

\subsection{Ship Model}

The aircraft carrier under study is a LHD (Landing Helicopter Dock) ship type which includes a ski-jump ramp to facilitate take-off operations [14] and a superstructure where bridge, radomes, antennas, stacks and other systems are installed $[4,7]$. The ship model was made of wood and built at a 1:100 geometric scale to avoid wind tunnel blockage effects. As the platform simulates the sea surface, the model only represents the ship structure above the waterline whose dimensions are indicated in Figure 2.

The non-symmetry of the ship means that airplanes would deal with 3D effects. Hence, three sections along the spanwise of the ski-jump ramp were studied to provide information to be applied in CFD validation process.

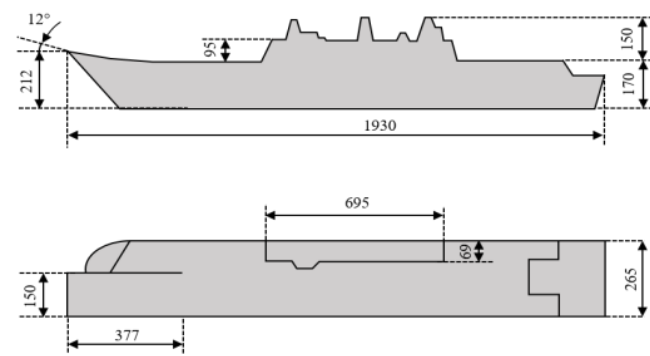

Figure 2: Aircraft carrier model dimensions (in millimetres).

\section{Particle Image Velocimetry}

Particle image velocimetry (PIV) is a non-intrusive technique that measures fluid flow providing instantaneous velocity fields. The high accuracy [20], the ability to obtain quantitative information and the capability to make global velocity measurements have elevated PIV to a special status in fluid mechanics [24]. Regarding the good results that PIV provides for turbulent flow studies [20], the flow structure due to the ski-jump ramp presence was analysed using this technique.

PIV equipment is constituted basically by the following components [24]: a system to generate particles with the required characteristics to seed the flow under study; a light source with enough energy to illuminate the region of interest; a CCD (chargecoupled device) camera to capture the light scattered by those particles; a synchronizer to control the trigger of all devices; and a software to process the images and compute the velocity vectors from the tracer particles displacements. Notice that, the laser system needs a cooler equipment and a set of lens to generate the laser light sheet. Figure 3 shows the PIV equipment used for these experiments.
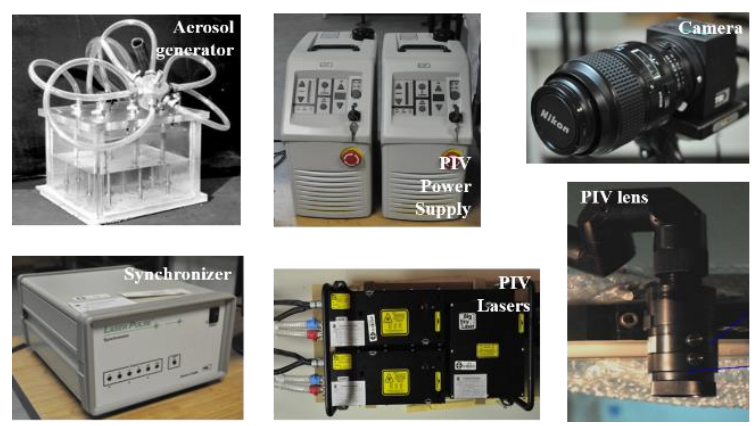

Figure 3: PIV equipment

As we can observe in Figure 1, the open test-section of the wind tunnel allows to capture the flow information about the region of interest. A Laskin nozzle atomizer [25] was used to obtain olive oil tracer 
particles with a diameter of the order of $1 \mu \mathrm{m}$ [26]. The characteristics of these tracer particles allow to scatter enough light and to avoid buoyancy and inertial problems. In addition, the seeding system is located downwash the test section, so the particles follow the closed-circuit and have enough time to be adapted to the flow velocity conditions.

A neodymium-doped yttrium aluminum garnet (Nd:YAG) laser was used to illuminate the particles transported by the flow. Two $190 \mathrm{~mJ}$ laser pulses were delivered, with a time separation $(\Delta \mathrm{t})$ of $25 \mu$ s between them, to compute the velocity components by avoiding blurred particle images. The data rate acquisition of PIV images was $10 \mathrm{~Hz}$ (10 pairs of images per second).

The images were recorded by a high-resolution CCD camera $(2048 \times 2048$ pixels $)$ that captures the light scattered by the tracer particles and an AF Nikkor 80200 camera lens. Notice that, the light laser sheet is located parallel the CCD sensor to avoid misalignment problems.

The synchronizer is in-charged to control the laser pulse and the camera exposure times ensuring that the camera records the flow information at the moment in which the laser is illuminating the flow. This parameter selection is introduced in the computer located in the control room where the different systems are connected.

The dimensions of the captured images are based on the camera field of view (FOV). For this experiment, a FOV of $250 \times 250 \mathrm{~mm} 2$ was obtained by calibrating the FOV with a standardized mesh located inside the laser light sheet. With the aim to fully capture upstream flow information and recirculation bubble region, two images (A and B) were assembled providing one map at each section (Figure 4). The reference axes were selected taking into account the Field of View (FOV) dimensions and region of interest. The image assembly was developed using an in-house Matlab code. The overlapped region reduces slightly the total dimensions of the image. In addition, three sections of the ski-jump ramp were studied to provide 3D information. The nonsymmetry of the structure implies that the selected regions to be studied are S1 (25\%), S2 (50\%) and S3 (75\%) of the ski-jump ramp spanwise (see Figure 4 ).
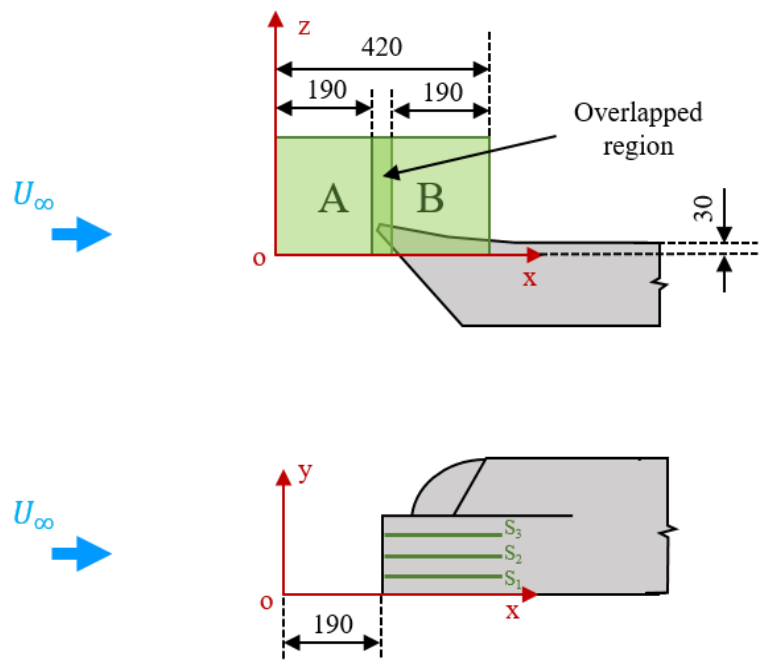

Figure 4: Ski-jump ramp dimensions and sections studied.

The velocity vector maps were obtained by using two sequential captures in the same area with a time separation $(\Delta \mathrm{t})$ of $25 \mu \mathrm{s}$. These images are subsampled via an interrogation window scheme. The selected size of the interrogation window was $32 \times 32$ pixels [24] with a $50 \%$ overlapping, following the Nyquist sampling criteria [27], as Figure 5 shows. The light scattered by the tracer particles is received by the CCD sensor that provides images in a digital format. Considering the time separation, the velocity vectors are computed in each interrogation window by determining the averaged particles displacement. 


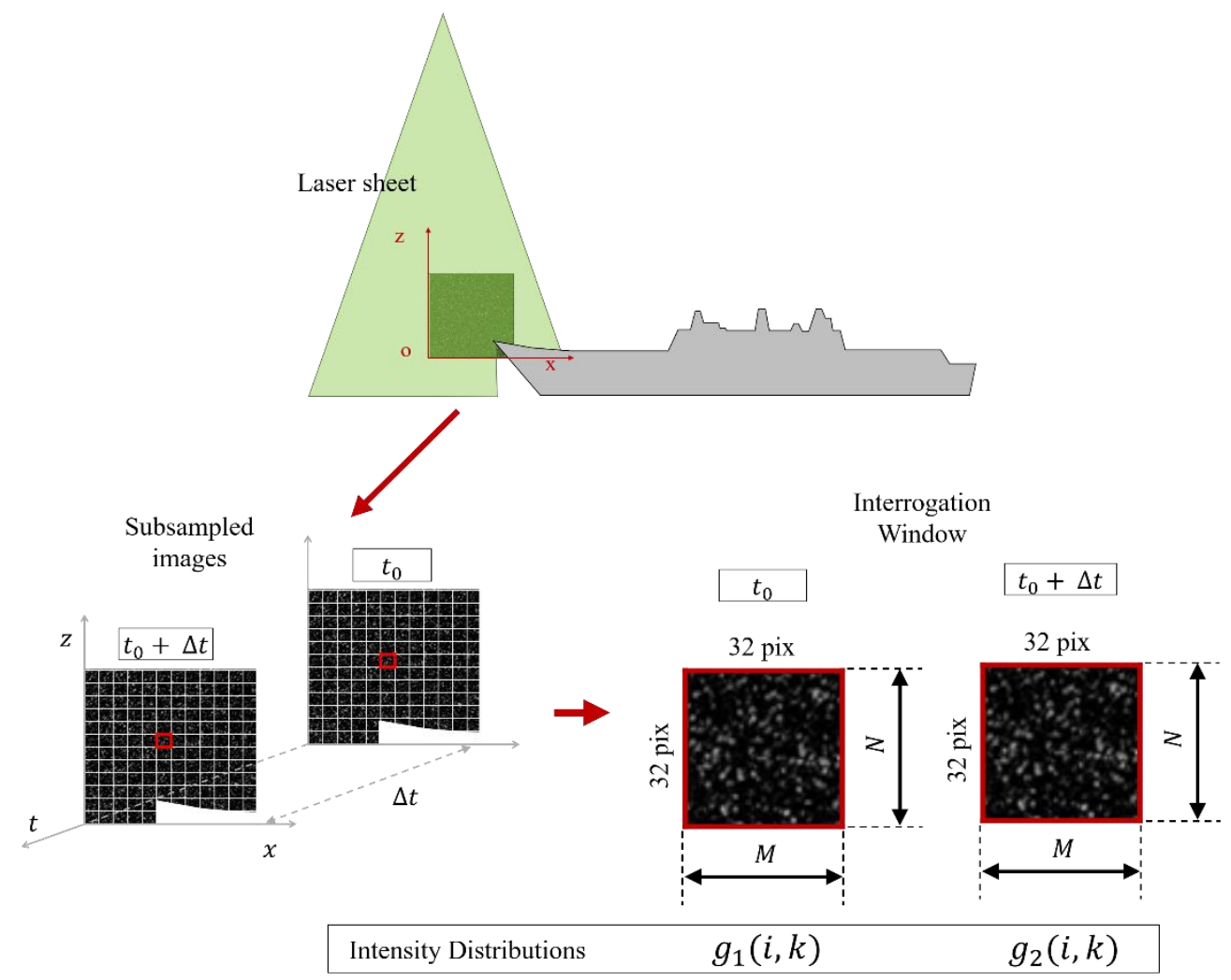

Figure 5: Interrogation window example.

The particle displacement is computed by using the statistical techniques based on cross-correlation as Figure 6 indicates. The maximum value of this crosscorrelation shows a strong peak displaced from the origin corresponding to the averaged motion of the particles contained within the interrogation window. This correlation peak was located with sub-pixel accuracy by fitting a Gaussian curve [28].

In the last years, the process to compute the crosscorrelation algorithm has been accelerated by means of advanced computational methods [27]. The most important feature of actual implementation of digital PIV (DPIV) technique is the use of 2D fast Fourier transform (FFT) to simplify and speed up the computational process [24]. Taking into account the similarity between spatial cross-correlation and convolution, the cross-correlation of two functions $\mathrm{g} 1(\mathrm{x}, \mathrm{z})$ and $\mathrm{g} 2(\mathrm{x}, \mathrm{z})$ is,

$$
\begin{aligned}
\Phi_{\text {cross }}(x, z)=g_{1}(x, z) \circ g_{2}(x, z) \\
=g_{1}(x, z) \otimes g_{2}(-x,-z)
\end{aligned}
$$

Where $\phi_{\text {cross }}(\mathrm{x}, \mathrm{z})$ is the cross-correlation function, o is the cross-correlation operator and $\otimes$ is the convolution operator.

Regarding Fourier transform properties,

$$
\begin{aligned}
& \left.\mathcal{F}\left[g_{1}(x, z) \otimes g_{2}(-x,-z)\right]=G_{1}(\zeta, \eta) \cdot G_{2}(-\zeta,-\eta)\right] \\
& \left.\mathcal{F}\left[g_{2}(-x,-z)\right]=G_{2}(-\zeta,-\eta)=G_{2} *(\zeta, \eta)\right]
\end{aligned}
$$

Where $\mathrm{G}_{2} *$ is the complex conjugate function of $\mathrm{G}_{2}$ and $\zeta, \eta$ are the components in the correlation plane.

Hence, the particle displacement can be obtained using equation 4.

$$
\Phi_{\text {cross }}(x, z)=\mathcal{F}^{-1}\left[G_{1}(\zeta, \eta) \cdot G_{2} *(\zeta, \eta)\right]
$$


Where $\mathcal{F}^{-1}$ is the inverse Fourier transform and $\mathrm{G}_{1}$ and $G_{2}$ are the Fourier transforms of the intensity distributions of each pair of images as Figure 6 shows.
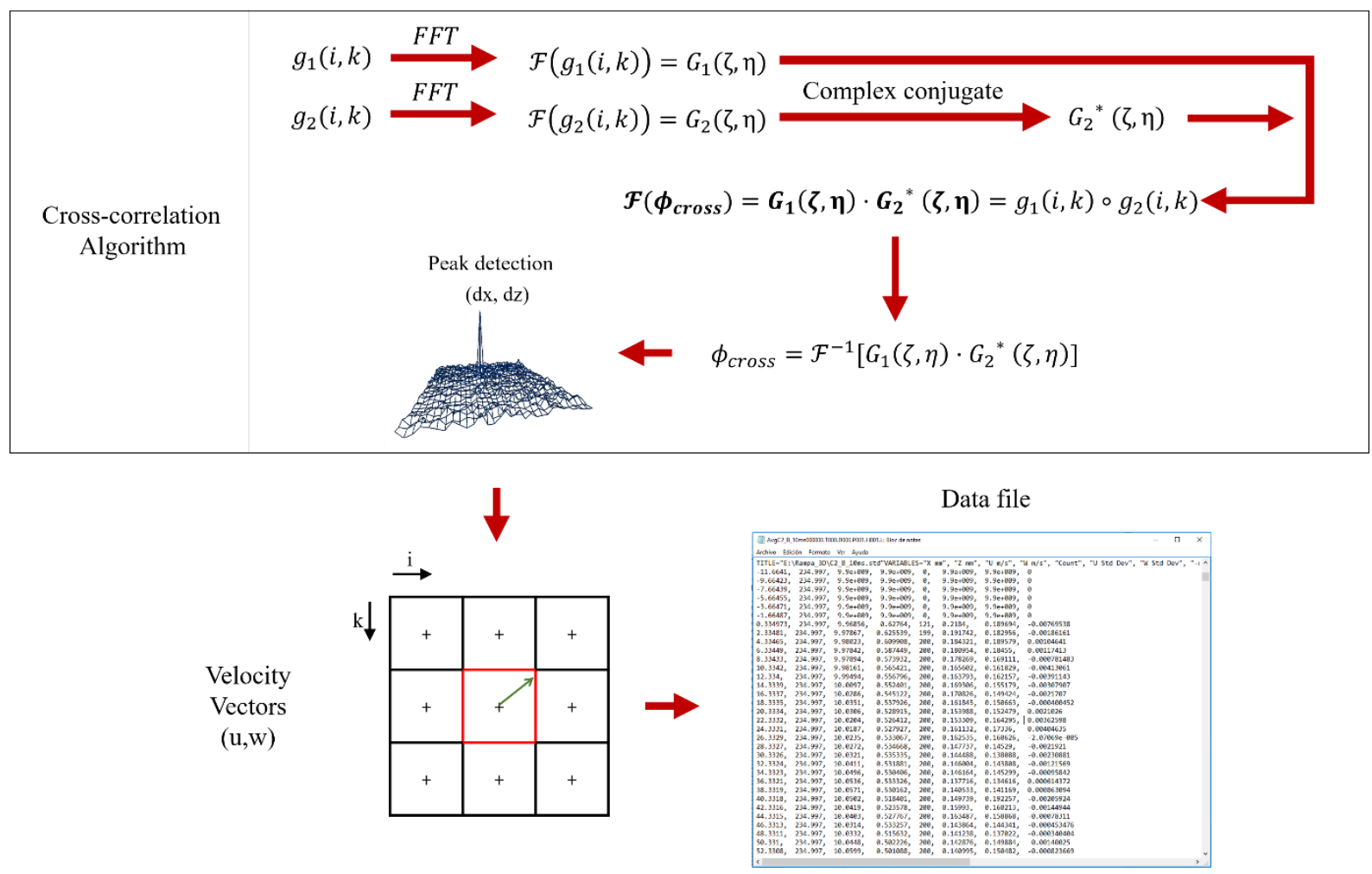

Figure 6: Cross-correlation process and results file.

Regarding the data file organization and the experimental conditions ( $\Delta \mathrm{t}$, magnification...), the velocity vector coordinates are indicated for each centre of the interrogation window. This small regions can be related with the grid nodes for CFD. Hence, it is possible to perform a validation process by comparing the results obtained in both cases. From this validation, it is possible to compute the error obtained in CFD codes.

\section{Results}

As stated, three sections were tested along the spanwise of the ski-jump ramp. The information obtained from PIV experiments allowed to compute the non-dimensional velocity (Ū) using the velocity components $(\mathrm{u}, \mathrm{w})$ and the wind tunnel velocity $\left(\mathrm{U}_{\infty}\right)$ at each case condition, as equation 5 indicates.

$$
\bar{U}=\left[\left(u^{2}+w^{2}\right)^{0.5}\right] / U_{\infty}
$$

Turbulence intensity (TI) maps were obtained from velocity standard deviation components $\left(\sigma_{\mathrm{u}}, \sigma_{\mathrm{w}}\right)$.

$$
T I=\left[\left(\sigma_{u}^{2}+\sigma_{w}^{2}\right)^{0.5}\right] / U_{\infty}
$$

Figure 7.a shows the non-dimensional velocity maps at the three sections. In addition, the streamlines were also represented to provide velocity flow structure and recirculation bubble configuration. The turbulence intensity maps are plotted in Figure 7.b. 

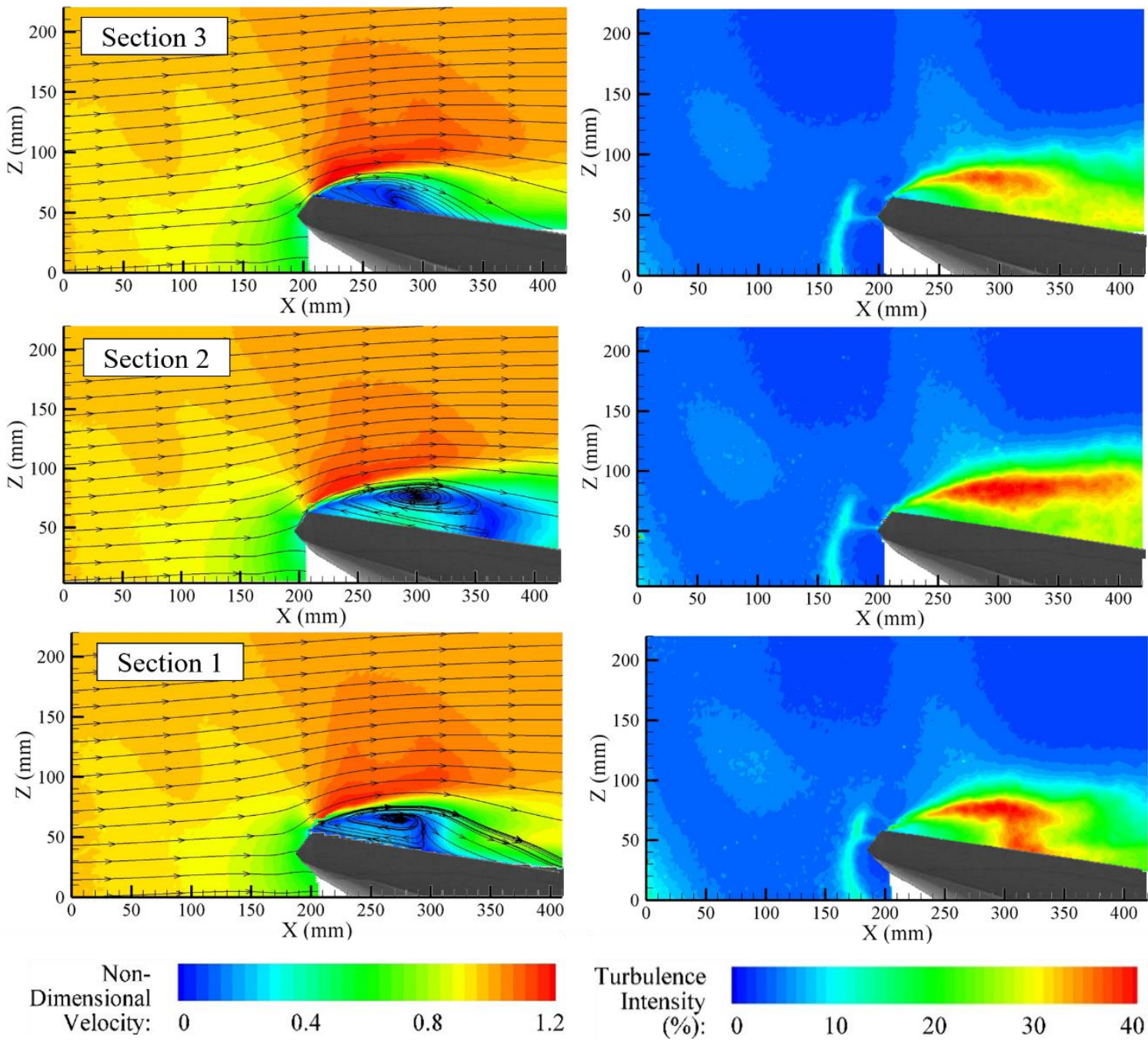

Figure 7: PIV maps at different sections of the ski-jump ramp (a) non-dimensional velocity (b) turbulence intensity.

Notice that, the lateral effects reduce the low velocity region close to the edges of the ski-jump ramp with respect to the central section (S2). It might be related with the non-symmetry of the ship, which also affects to the flow configuration resulting in a lower recirculation bubble height at the port of the ship. However, the length of the bubble at section 1 is bigger than in section 3. As it can be observed, the incoming flow is slightly affected upstream by the ski-jump ramp presence.

Regarding turbulence intensity (see Figure 7.b), the central section has a bigger turbulent shear layer than the lateral ones. In addition, section 1 presents a remarkable structure difference which could be due to the sharp lateral edge. However, section 3 has an intensity value reduction which can be related to the sponson presence which is characterized by a curved edge that might reduce the lateral effects.

Figure 8 shows the location of reattachment point and the recirculation bubble centre in $x-y$ plane. The reattachment point is defined as the point where the velocity direction over the ski-jump ramp changes and is equal to zero [29]. The recirculation bubble centre is the point where the velocity is zero inside the recirculation bubble region and where streamlines collide. Notice that, y-axis is non-dimensionalized 
using $\mathrm{b}$ parameter which is the spanwise size. The representation of these variables allow to show the lateral effect over the recirculation bubble configuration. Both variables are not directly related although lateral reduction is observed. Despite the high reduction observed in section 3 , the reattachment point location for section 1 is lower. The recirculation bubble centre at both sides is located at same horizontal position.

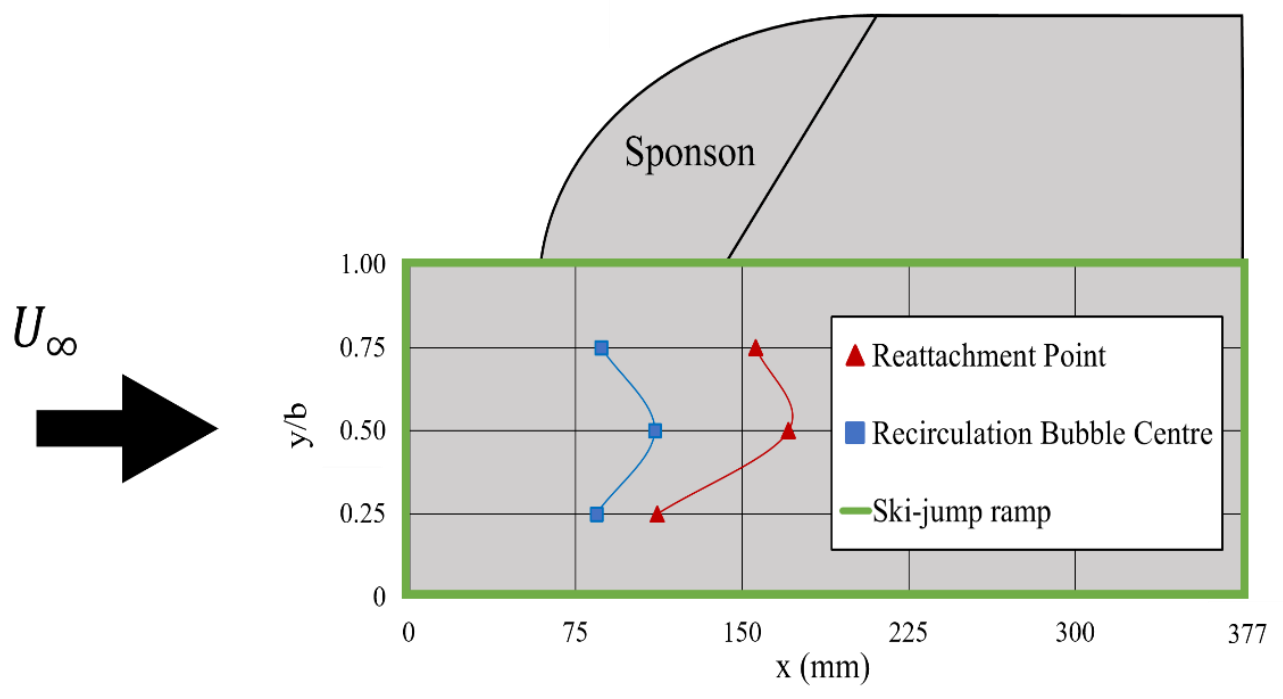

Figure 8: Recirculation bubble centre and reattachment point.

\section{Conclusions}

The short runway available in aircraft carriers implies the need of additional systems to help aircraft to take-off as the ski-jump ramp that increases the aircraft lift resulting in successful take-off operation. Ahead wind condition is the standard configuration during aircraft performances. The ski-jump ramp presence at this wind condition generates aerodynamic adverse effects for aircraft operations and consequently, pilots have to handle with workload increment and low safety levels during on board operations. Hence, the study of this problem has become more common by using wind tunnel tests and computational methods. Moreover, particle image velocimetry technique has shown to be a powerful tool in the investigation of this kind of flows. In addition, PIV presents useful results for CFD validation.

Experimental tests presented in this papers have been carry out in the facilities of INTA. A closed-circuit wind tunnel with an open test section was used to perform PIV tests. The aircraft carrier model presents a non-symmetric structure which means that aircraft would deal with $3 \mathrm{D}$ effects. Three sections along the spanwise were studied to provide 3D information. Furthermore, two PIV captures at each section were developed to provide upstream information of the incident flow.

The results have shown the presence of a 3D flow structure formed by a recirculation bubble located above the ski-jump ramp and bounded by a highly turbulent and non-stationary shear layer. The recirculation bubble has different configuration at each section under study proving three-dimensional nature of the flow over the ramp. Furthermore, the lateral sides are affected by different structures, so the recirculation bubble changes in each section. Turbulence intensity maps also provide turbulent shear layer variations in size. In addition, section 1 presents a region with high turbulence values that might be related with the lateral structure. 
PIV maps were analysed to study the centre of the bubble vortex height and the reattachment point of the bubble. Results showed higher values in the central section (S2). Values of these variables in the port section (S1) are lower and they show a slightly asymmetry with respect to the values measured in starboard section (S3).

Finally, we can conclude that the aerodynamic flow in the aircraft carrier ski-jump was experimentally investigated by means of particle image velocimetry (PIV). The results have demonstrated the capability of PIV resolving the 3D flow structure developed over the ski-jump ramp and providing insight and data to be used in the validation process of CFD numerical codes.

\section{References}

[1] Maslov, L. A., Valuev, N. O., \& Zharinov, A. V. (1999). The experience of aerodynamic disturbances research behind an aircraft-carrier ship with elements for safe operation of ship-based aircraft. In RTO Meeting proceedings (pp. 9-1).

[2] Kääriä, C. H., Wang, Y., White, M. D., \& Owen, I. (2013). An experimental technique for evaluating the aerodynamic impact of ship superstructures on helicopter operations. Ocean Engineering, 61, 97-108.

[3] Wadcock, A., Yamauchi, G., Solis, E., \& Pete, A. (2011, June). PIV measurements in the wake of a full-scale rotor in forward flight. In 29th AIAA Applied Aerodynamics Conference (p. 3370).

[4] Stargel, D., \& Landman, D. (2012, January). A wind tunnel investigation of ship airwake/rotor downwash coupling using design of experiments methodologies. In 50th AIAA Aerospace Sciences Meeting including the New Horizons Forum and Aerospace Exposition (p. 767).

[5] Swales, C., et al. "LDV measurements above the flight deck of a model frigate." AIAA, Aerospace Sciences Meeting \& Exhibit, 35th, Reno, NV. 1997.

[6] Maslov, L. A., Valuev, N. O., \& Zharinov, A. V. (1999). The experience of aerodynamic disturbances research behind an aircraft-carrier ship with elements for safe operation of ship-based aircraft. In RTO Meeting proceedings (pp. 9-1).

[7] Czerwiec, Ryan M., and Susan A. Polsky. "LHA air wake wind tunnel and CFD comparison with and without bow flap." AIAA 4832 (2004): 2004.

[8] Lee, R. G., \& Zan, S. J. (2002, September). Wind tunnel testing to determine unsteady loads on a helicopter fuselage in a ship airwake. In ICAS 2002 Congress (pp. 3111-1).

[9] Swales, C., \& Breeze, G. (1997, January). LDV measurements above the flight deck of a model frigate. In 35th Aerospace Sciences Meeting and Exhibit (p. 712).

[10] Bardera-Mora, R., Barcala-Montejano, M. A., RodríguezSevillano, A., de Diego, G. G., \& de Sotto, M. R. (2015). A spectral analysis of laser Doppler anemometry turbulent flow measurements in a ship air wake. Proceedings of the Institution of Mechanical Engineers, Part G: Journal of Aerospace Engineering, 229(12), 2309-2320.

[11] Bardera-Mora, R., León Calero, M., \& García-Magariño, A. (2017). Aerodynamic effect of the aircraft carrier island on flight deck flow with cross wind. Proceedings of the Institution of Mechanical Engineers, Part M: Journal of Engineering for the Maritime Environment, 1475090216689172.

[12] Nacakli, Y., Landman, D., \& Doane, S. (2012). Investigation of Backward-Facing-Step Flow Field for Dynamic Interface Application. Journal of the American Helicopter Society, 57(3), 1-9.

[13] Brownell, C. J., Luznik, L., Snyder, M. R., Kang, H. S., \& Wilkinson, C. H. (2012). In situ velocity measurements in the near-wake of a ship superstructure. Journal of Aircraft, 49(5), 1440-1450.

[14] Hernando, J. L., \& Martínez-Val, R. (2012). “Carrier suitability of land-based aircraft". In 28th International Congress of the Aeronautical Sciences (ICAS).

[15] Bardera-Mora, R., Barcala-Montejano, M.A., RodríguezSevillano, A., Nova-Trigueros, J. "Passive flow control over the ski-jump of aircraft carriers." Ocean Engineering 114 (2016): 134-141.

[16] Polsky, Susan A. "CFD prediction of air wake flow fields for ships experiencing beam winds." AIAA paper 3657 (2003): 21.

[17] Zhang, H., Prasad, J., \& Mavris, D. (1994, August). Ship airwake effects on the helicopter rotor aerodynamic loads. In 19th Atmospheric Flight Mechanics Conference (p. 3509).

[18] Brownell, C., Luznik, L., Snyder, M., \& Kang, H. S. (2012, June). Velocity measurements in a ship airwake with crosswind. In 42nd AIAA fluid dynamics conference and exhibit (p. 2851).

[19] Polsky, S. A., \& Ghee, T. A. (2008). Application and verification of internal boundary conditions for antenna mast wake predictions. Journal of Wind Engineering and Industrial Aerodynamics, 96(6), 817-830.

[20] Kompenhans, J., Raffel, M., \& Willert, C. (1996). PIV applied to aerodynamic investigations in wind tunnels, von Karman Institute for Fluid Dynamics, Lecture Series 1996-03. Particle Image Velocimetry, Rhode-St-Genese (Belgium). 
[21] Roosenboom, Eric WM, Arne Stürmer, and Andreas Schröder. "Comparison of PIV measurements with unsteady RANS calculations in a propeller slipstream." Proceedings of 27th AIAA Applied Aerodynamics Conference. 2009.

[22] Polsky, S. (2003, June). CFD prediction of airwake flowfields for ships experiencing beam winds. In 21st AIAA Applied Aerodynamics Conference (p. 3657).

[23] Lumsden, R. B. (2003). Ship air-wake measurement, prediction, modelling and mitigation. DSTL/TR06951. Defence Science and Technology Laboratory, UK.

[24] Prasad, A. K. (2000). Particle image velocimetry. CURRENT SCIENCE-BANGALORE-, 79(1), 51-60.

[25] Echols, W. H., \& Young, J. A. (1963). Studies of portable air-operated aerosol generators (No. NRL-5929). NAVAL RESEARCH LAB WASHINGTON DC.

[26] Kähler, C. J., Sammler, B., \& Kompenhans, J. (2004). Generation and control of tracer particles for optical flow investigations in air. In Particle Image Velocimetry: Recent Improvements (pp. 417-426). Springer Berlin Heidelberg.

[27] Willert, C. E., \& Gharib, M. (1991). Digital particle image velocimetry. Experiments in fluids, 10(4), 181-193.3

[28] Raffel, M., Willert, C. E., Wereley, S. T., \& Kompenhans, J. (2007). Particle Image Velocinmetry: A Practical Guide Experimental Fluid Mechanics. 2nd Revised Edition.

[29] Hasan, M. A. Z., 1992. The flow over a backward-facing step under controlled perturbation: laminar separation. Journal of Fluid Mechanics, 238, 73-96. 